\title{
PHASE-SEPARATION PHENOMENA IN SOLUTIONS OF POLY(2,6-DIMETHYL-1,4-PHENYLENE OXIDE). III. PULSE-INDUCED CRITICAL SCATTERING OF SOLUTIONS IN TOLUENE
}

\author{
D. M. KOENHEN and C. A. SMOLDERS
}

Department of Colloid and Interface Science. Twente Unicersity of Technology. Enschede. The Netherlands

\author{
M. GORDON \\ Institute of Polymer Science. Unicersity of Essex, Wivenhoe Park, \\ Colchester CO 4.3S Q. U.K.
}

\section{SYNOPSIS}

For the polymer-solvent system poly(phenylene oxide) in toluene the mechanism and kinetics of crystallization have been studied with the Pulse Induced Critical Scattering technique. It was found that after a delay-time $r$ the growth mechanism was diffusion controlled. The delay-time is thought to be connected with the nucleation of the crystallites and it disappeared in the "sceded" crystallizations studied. After incomplete melting of crystallites the first stages of growth resemble a condensation reaction.

\section{INTRODUCTION}

With the Pulse Induced Critical Scattering (PICS) method it is possible to follow isothermally the light scattering of polymer solutions. which is induced by a preceding thermal pulse (in most instances this means a cooling step). The application of this technique in the determination of the phase separation phenomena in liquid-liquid demixing systems has been reported [1]. The system poly(2,6-dimethyl-1,4-phenylene oxide) (PPO resin: a registered tradename of General Electric $\mathrm{Co}$.) in toluene exhibits a phase separation on cooling which has been shown to be a crystallization phenomenon [2]. It was found that the crystallization occurs after a delay-lime which has been measured by optical microscopy [3]. This delay-time is sometimes attributed to the time necessary for the growing centers to reach detectable dimensions [4]. The classical way in which these delay-times and the kinetics of crystallization can be measured is by dilatometry. This method, however, is not very sensitive in the first stages of crystallization. An advantage of the dilatometry method is that the crystallization can be followed to completion [5]. With the purpose to improve the limit of detection to the smallest particles (the nuclei), we have used the PICS type light scattering apparatus as developed by Gordon and co-workers [6]. 
This light scattering technique enables one to detect particles which are smaller than the wavelength of the light used. By making a few assumptions, the kinetics in the very first stages of the phase separation can be determined as well. Because of the very small sample size used in the PICS technique, assuring that rapid temperature equilibration is reached, even very fast crystallizations can be monitored.

\section{THEORETICAL CONSIDERATIONS}

\section{Light Scattering}

The light scattering of particles with small diameters compared with the wavclength of the incident light is given by Rayleigh [7] as

$$
i_{\theta}=\frac{8 \pi^{2}}{r^{2} \lambda^{\prime 4}} \nu \alpha^{2} I_{0}\left(1+\cos ^{2} \theta\right)
$$

where $i_{\theta}$ is the light scattered at angle $\theta ; r$ is the distance to scattering volume; $\lambda^{\prime}$ is the wavelength of light used, divided by the refractive index of the medium; $\nu$ is the number of particles; $\alpha$ is the polarizability of the particles; and $I_{0}$ is the intensity of the incident light.

The light scattered at an angle $\theta$ thus depends on the square of the polarizability $\alpha$. The polarizability $\alpha$ increases linearly with the volume. This means that, taking the particles as spherical, the intensity of the scattered light varies with the sixth power of the radius [7]. When the particles are larger but remain smaller than the wavelength, the light scattered at larger angles decreases with a factor $P(\theta)$. This type of scattering is known as Debye scattering.

For isotropic spheres this factor is

$$
P(\theta)=\left[\frac{3}{U^{3}}(\sin U-U \cos U)\right]^{2}
$$

with $U=2 \pi\left(L / \lambda^{\prime}\right) \sin (\theta / 2)$ and $L$ being the diameter of the sphere.

Equation (2) shows that when the spherical particle is growing, the dissymmetry of intensities measured at two angles (e.g., $30^{\circ}$ and $90^{\circ}$ ) increases rapidly. So it is easy to determine which kind of scattering is being followed by monitoring the dissymmetry of the scattered light. This is important in order to determine the order of magnitude of the dimensions of the particles which one observes. From the measurements of the dissymmetry in Debye scattering one could, in principle, determine the radius of the particles.

\section{Kinetics of Crystallization}

The most widely used equation to describe the kinetics of crystallization from polymer melts is the Avrami-Evans equation [5].

$$
X=1-\exp \left(-k t^{n}\right)
$$

where $X$ is the degree of conversion; $k$ is the rate constant; $t$ is time; and $n$ is the Avrami exponent. 
For very small conversions the first term of a series expansion of eq. (3) can be used giving $\mathrm{X}=k t^{n}$.

For the case of growth from a solution the kinetics of heterogeneous crystallization have been worked out mathematically by Turnbull [8]. For low conversions his equations reduce to a form equivalent to eq. (3), with $n=3 / 2$ for diffusion controlled growth and $n=3$ for interface controlled growth and assuming for both types a fixed number of nuclei. When nuclei are generated during the crystallization the exponent shifts to a slightly higher value.

Hence if heterogeneous nucleation is assumed with a fixed number of nuclei the Rayleigh light scattering at small $t$ values in the case of diffusion-controlled growth should depend on the third power of time (since for small conversions $I$ is proportional to $X^{2}$ ). For interface-controlled growth Rayleigh light scattering is proportional to the sixth power of time (see Table I).

Oster [9] has considered the light scattering during various types of polymerization reactions. In some instances the crystallization from a solution could be considered as a kind of polymerization reaction. Oster finds for a condensation reaction that the light scattering is proportional to time. The light scattering in radial polymerization depends quadratically on time.

\section{EXPERIMENTAL}

The experiments were performed with a PPO sample characterized by $M_{n}$ $=25,000$ and $M_{w}=42,000$ (by gel permeation chromatography).

The toluene used was of analytical grade. Stock solutions of different concentrations were made, from which the capillaries for the PICS instrument were filled with a syringe and sealed. From these stock solutions, also, the dilatometric measurements were made. using a digital density meter DMA 50 from Anton Paar. This apparatus measures density changes as small as $10^{-5} \mathrm{~g} / \mathrm{cm}^{3}$.

The PICS instrument has been described in the literature $[1,6]$. The light scattered by the polymer solution after a fast temperature step is recorded at angles of $30^{\circ}$ and $90^{\circ}$. The laser used as a light source was a $\mathrm{He} / \mathrm{Ne}$ type (Spectra Physics 134).

\section{RESULTS}

The light scattering curves obtained with PICS after cooling a homogeneous solution (which was heated for $10 \mathrm{~min}$ at a temperature of $20^{\circ} \mathrm{C}$ above the crystalline melting point of that solution [10]) all show a long delay-time after

TABLE

\begin{tabular}{|c|c|c|c|c|}
\hline \multirow{2}{*}{$\begin{array}{c}\text { Growth } \\
\text { Controlled }\end{array}$} & \multicolumn{2}{|c|}{$\begin{array}{c}\text { Heterogeneous } \\
\text { Nucleation }\end{array}$} & \multicolumn{2}{|c|}{$\begin{array}{c}\text { Homogeneous } \\
\text { Nucleation }\end{array}$} \\
\hline & $\begin{array}{l}n=3 / 2 \\
n=3\end{array}$ & $\begin{array}{l}q=3 \\
q=6\end{array}$ & $\begin{array}{l}n=3 / 2 \text { to } 2 \\
n=3 \text { to } 4\end{array}$ & $\begin{array}{l}q=3 \text { to } 4 \\
q=6 \text { to } 8\end{array}$ \\
\hline
\end{tabular}

- $n$ is the Avrami exponent, $q$ is the expected scattering exponent, $I \sim t^{q}$, for Rayleigh scattering and small conversions. 


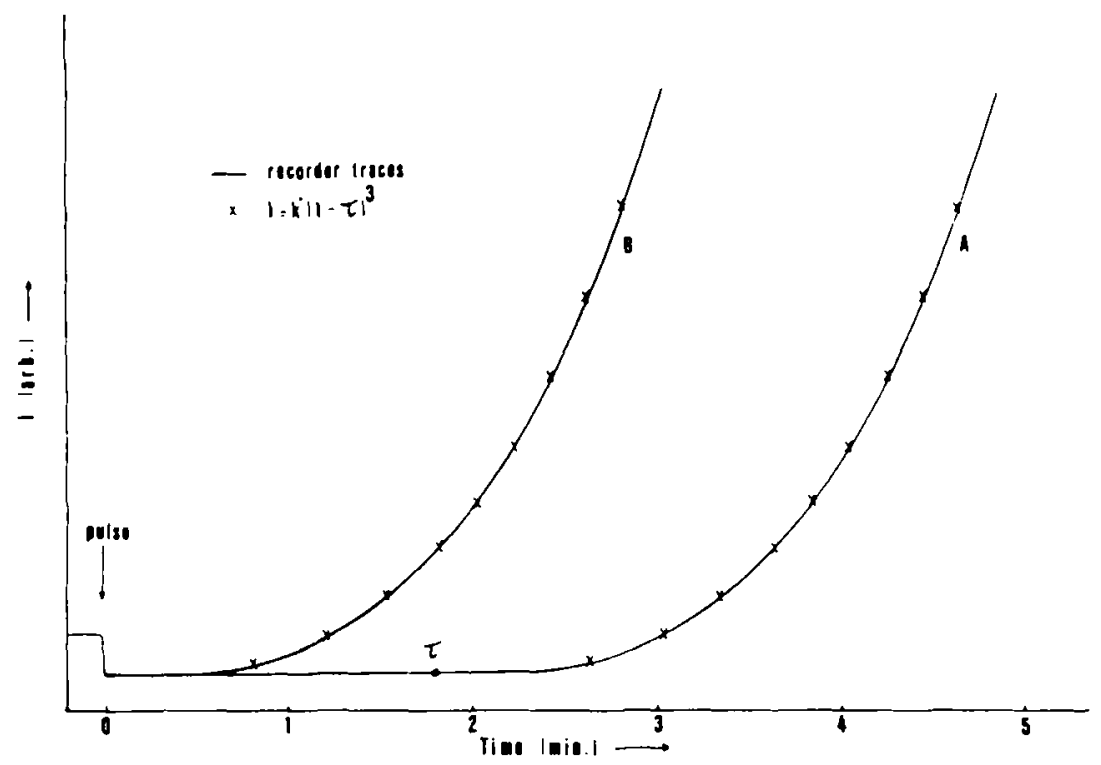

FIG. 1. PICS light scattcring curves (recorder traces) for a 20\% PPO solution in toluene. Pulse temperature to $25^{\circ} \mathrm{C}$. (..-) Theoretical, $n=1.5 ;(-)$ measured.

which scattering increases. In analyzing the curves care is taken to use only the scattering in the first stages of the crystallization process, up to scattering intensities less than one third of that observed at the inflection point, as was ascertained by control experiments. In these curves there is no additional dissymmetry detectable which could be attributed to the growth of larger particles. The scattering curves detected at $30^{\circ}$ and $90^{\circ}$ are equivalent. It can therefore be concluded that Rayleigh scattering is observed.

A typical light scattering curve of this type is curve A in Figure 1. When a phase-separated solution is not heated at high enough temperatures (e.g., to a temperature in the region of the high end of the DSC (differential scanning calorimetry) melting endotherm) then after cooling curve B can be obtained. The form of the light scattering curve is the same as in curve A, but the delaytime has disappeared. Thus the assumption was made that the growth of particles is the same in both experiments but in curve $A$ there is a time lag, necessary for nucleus formation. Curve B is, therefore, interpreted as a "seeded" crystallization. Curve B could be described by a third power law. Since in curve A the growth part is identical to curve $B$ this curve can be described by

$\tau$ is the delay time.

$$
\begin{array}{ll}
I=k^{\prime}(t-\tau)^{3} & \text { at } t \geq \tau \\
I=0 & \text { at } t<\tau
\end{array}
$$

This equation gives a satisfactory fit in all experiments. Without assuming a delay-time, higher exponents were obtained with deviations, most notably in the first part of the curve. In Figure 2 the delay-times, obtained after fitting the curves with eq. (4), are presented as a function of temperature and concentration. 


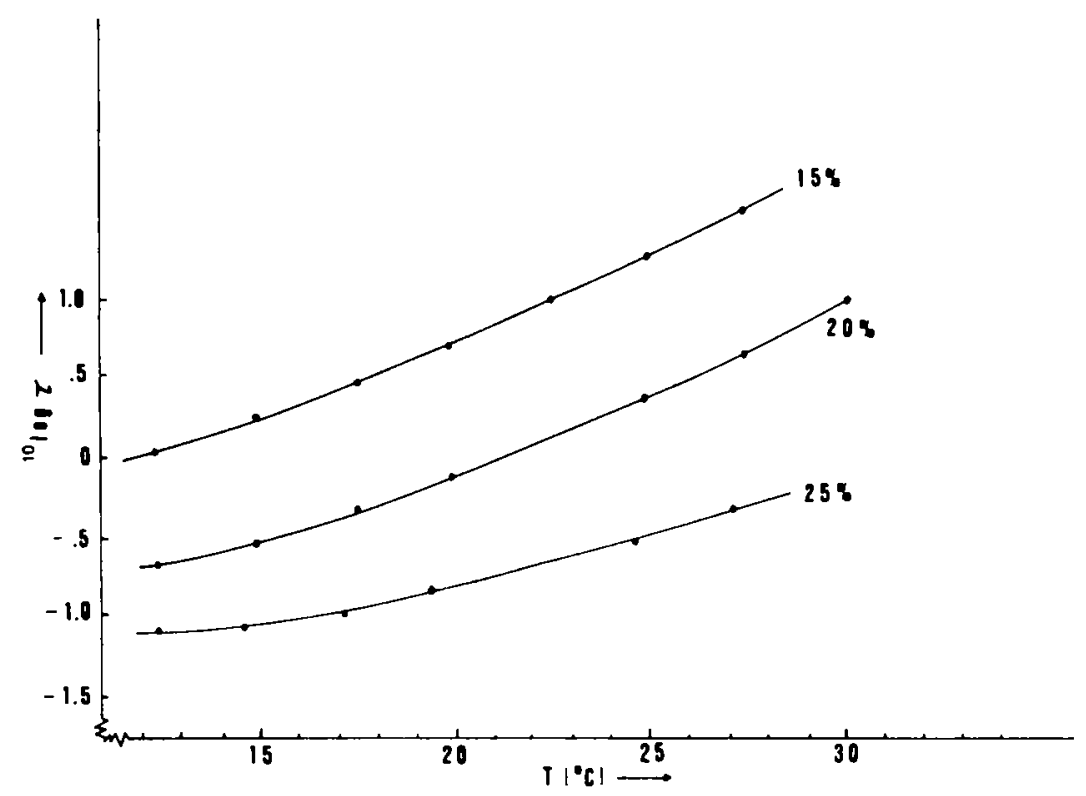

FIG. 2. Delay-time measured by PICS for solutions of PPO in toluenc. $\tau$ is given in minutes.

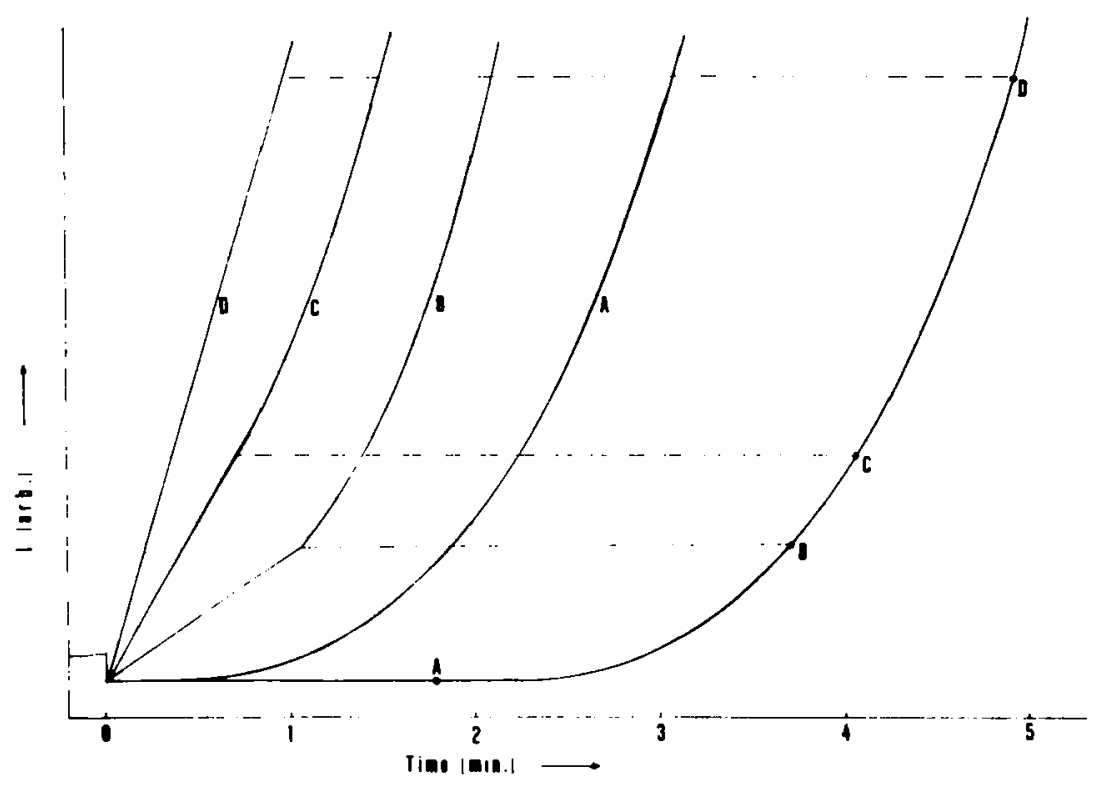

FIG. 3. PICS curves for seeding experiments on a $20 \%$ PPO solution in toluene. Capitals refer to original extent of crystallization as indicated on the outer right curve. Pulses to $25^{\circ} \mathrm{C}$. intermediate heating to $60^{\circ} \mathrm{C}$ for $30 \mathrm{~min}$.

If a phase separation is brought about to different degrees of crystallization, and the separated solution is heated until the region in which partial melting occurs, some interesting phenomena in the field of seeded crystallization can be observed. For the experiments in Figure 3 the temperature of heating, after 


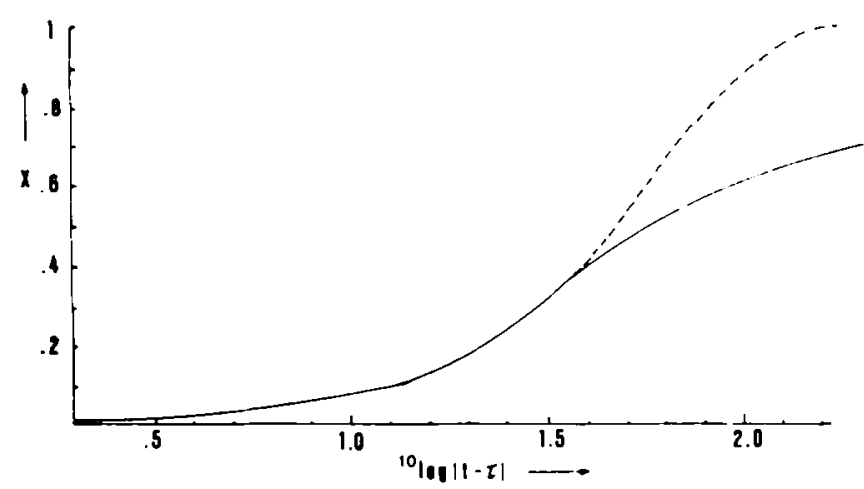

FIG. 4. Density measurements with DMA 50 for $20 \%$ solution of PPO in toluene at $30^{\circ} \mathrm{C}$. The theorctical curve (dashed line. $n=1.5$, with $\tau=0$ ) has been shifted horizontally to obtain coverage of the experimental curve (solid line), which has been plotted with the delay-time ( $r=7 \mathrm{~min}$ ) found by PICS.

the initial phase separation, was kept constant, as was the time of this heating. The difference is in the extent of phasc scparation before heating.

Finally, in Figure 4 a typical curve obtained by density measurements is shown.

\section{DISCUSSION}

The density measurements of the crystallizing solution give a crystallization isotherm which at low conversions cannot be described by the normal Avrami-Evans equation. However, when the delay-time is introduced the Avrami exponent can be computed as $n=1.5 \pm 0.1$ (Fig. 4). This conclusion agrees with the measurements of heterogencous crystallization of PPO from the melt as assessed by Packter and Sharif [11]. Here also a delay-time was found and an Avrami exponent of $n=1.6$. The exponent $n=1.5$ can also be deduced from the measurements by optical microscopy of Van Emmerik and Smolders [3], who found that the radii of the spheres growing in the solution increased with the square root of time (assuming a constant number of nuclei).

From the absence of additional dissymmetry during the first stages of growth as followed by PICS we conclude that in this stage we are observing Rayleigh scattering. It is known that the crystallinity in the final material is low [12]. The crystallization is possible here by solvent molecules which constitute part of the crystalline unit cell [13]. The high amorphous content of the small particles makes it reasonable to neglect anisotropy in the scattering particles. The scattering is thus thought to arise from the higher density of the spheres. Of course, in polymers which form more ideal crystallites, one should make a correction for anisotropy.

Thus from the density measurements where we found that $n=1.5$ we can expect that the light scattered will depend on $t^{3}$ after a certain delay-time. As is shown, this is precisely what is found in these solutions. 
The Avrami exponent found for these solutions shows that the growth is diffusion-controlled. This means that material is transported from bulk solution to the crystal surface. Since the overall crystallinity in the spheres is low, this suggests that only parts of the macromolecules are able to crystallize. The fraction of the chain which can participate in crystallization and the time it takes for its formation out of disordered chains, will, of course, be governed by the internal ordering ability of the macromolecular chain. The sequences of ordered segments have to diffuse towards each other. When after a previous phase separation the solution is heated for some time at a temperature in the region of the high end of the melting endotherm, as found by DSC [12], the delay-time has disappeared. This type of crystallization can be explained as a "seeded" crystallization.

When the melting procedure is less rigorous (viz., not long enough) we obtain, after pulsing back to $20^{\circ} \mathrm{C}$, a linear increase in scattering intensity at first. After passing the point to which the original crystallization had proceeded, a normal curvature takes over. The slope of the initial part of these curves is very much dependent on the original degree of crystallization. This effect can be explained by assuming incomplete melting of the crystallites and/or incomplete diffusion of crystallizable material from the area of the old crystallite into bulk solution. When this solution crystallizes again, crystallization probably occurs within the outline of the old crystallite.

Because of the availability of material necessary for growth within this area, crystallization resembles a condensation reaction. After the complete old volume has been crystallized, the normal growth situation (diffusion-controlled) is restored again. When the degree of conversion of the original crystallization is higher, the area in which this phenomenon can occur is larger and the slopes are steeper. This crystallization in molten crystallite volumes has been observed for spherulites of other materials as well.

In these experiments all of the original scattering had disappeared before the second temperature jump was made. This, together with the prolonged induction times for the completely molten solution suggests that the crystallization of PPO involves a two-step nucleation process. It is probable that first a conformation change is needed before nucleation and growth of crystals can occur. In the seeded experiments the crystals may have disappeared, but the material is still in the right conformation.

The experiments reported above are in agreement with DSC results reported earlier [12]. It is clear that the PICS light scattering technique can give very useful information about the first stages of nucleation from solution.

The authors thank Dr. J. Goldsbrough and Mr. B. W. Ready for carrying out part of the measurements.

\section{REFERENCES}

[1] K. W. Derham, J. Goldsbrough, and M. Gordon, J. Pure Appl. Chem., 38, 97 (1974).

[2] D. M. Koenhen and C. A. Smolders, J. Polym. Sci. Polym. Phys. Ed., in press.

[3] P. T. van Emmerik and C. A. Smolders, Eur. Polym. J.. 9. 931 (1973). 
[4] W. J. Dunning et al., Nucleation. A. C. Zettlemoyer, Ed., Marcel Dekker, New York. 1969.

[5] L. Mandelkern. Crystallization of Polymers. McGraw-Hill, New York. 1964.

[6] J. W. Kennedy, M. Gordon, and G. A. Alvarez. Polimery (Warsaw) No. 10. 1464 (1975).

[7] See, for instance. G. Oster, in Phusical Methods of Chemistry. Interscience, New York, 1972. pt. 3 A.

[8] D. Turnbull, Solid State Phy'sics, Vol. 3, Academic Press, New York, 1956.

[9] G. Oster, J. Colloid Sci., 2. 291 (1974).

[10] P. T. van Emmerik and C. A. Smolders. J. Polym. Sci.. C, 38.73 (1972).

[11] A. Packter and K. A. Sharif, J. Polym. Sci., B, 9. 435 (1971).

[12] D. M. Koenhen and C. A. Smolders, J. Polym. Sci. Polym. Phys. Ed. in press.

[13] E. P. Magré and J. Boon. Proc. IUPAC Int. Symp., Lciden, 1970. p. 835. 